\title{
Excellence in healthcare is celebrated at The BMJ Awards 2014
}

In this News story (BMJ 2014;348:g3210, doi:10.1136/bmj. g3210), we incorrectly listed South London and Maudsley NHS Foundation Trust as the location of the 3 Dimensions of Care for Diabetes project, when it is, in fact, King's College Hospital NHS Foundation Trust. This error also appears in The BMJ
Awards ceremony brochure, which is available as a "Data supplement" link in this News story.

Cite this as: BMJ 2014;348:g3319

๑ BMJ Publishing Group Ltd 2014 